\title{
Diagnostic Value of High Frequency Ultrasound on Pilomatricoma
}

\author{
Li Kaiwen, Zhang Qiang \\ Department of Ultrasound, Jingzhou Central Hospital, The Second Clinical Medical College, Yangtze University, Jingzhou, China
}

\section{Email address:}

likaiwen1234@163.com(Li Kaiwen)

\section{To cite this article:}

Li Kaiwen, Zhang Qiang. Diagnostic Value of High Frequency Ultrasound on Pilomatricoma. Science Journal of Clinical Medicine. Vol. 7, No. 1, 2018, pp. 9-12. doi: 10.11648/j.sjcm.20180701.12

Received: March 16, 2018; Accepted: March 28, 2018; Published: May 3, 2018

\begin{abstract}
To analyze the characteristics of high-frequency ultrasonography of pilomatricoma and discuss the value of high-frequency ultrasonography in the diagnosis of pilomatricoma, ultrasonographic data of 71 cases (77 patients) with pilomatricoma diagnosed by high-frequency ultrasonography and pathology in our hospital from June 2013 to June 2017 were retrospectively analyzed. The location, size, shape, border, echo and blood supply status of the sonographic images were summarized. As a Result, among the 71 patients (77), there were 62 cases located at the head and neck, 11 at limbs and 4 at the trunk. 66 cases were single and 5 cases were multiple. All the tumors were subcutaneously located. The typical characteristics of pilomatricoma: it is more common in young people. They are hypoechoic nodules on hypodermatic parts of head and neck. The boundary is clear. The shape is regular, accompanied by calcification within the visible dot-like blood flow signal. There is inner calcification within the visible dot-like blood flow signal. It is concluded that ilomatricoma has certain sonographic features. High-frequency ultrasonography has a significant impact in the diagnosis.
\end{abstract}

Keywords: Pilomatricoma, High-Frequency Ultrasound, Diagnostic Value

\section{Introduction}

Pilomatricoma, also known as calcifying epithelioma, is a benign tumor that is presented at the junction between the deep dermis and the subcutaneous fat. It originates from hair follicle stromal cells and rarely has malignant transformation [1] Hair mother tumor is common in young people. The history of the disease is usually long, often occurring in the head and neck. It is usually solitary, sometimes multiple. Because pilomatricoma has low incidence, clinical and imaging manifestations are diverse, clinical reports and imaging reports are scarce, and doctors' knowledge of them is different. All factors make clinical and imaging diagnosis uneven. Depending on the literature reports, the diagnostic coincidence rate is about $\mathrm{O} \sim 95.2 \%$ [2]. It is often misdiagnosed as subcutaneous calcium salt deposit, epidermoid cyst, calcified lymph node, hemangioma and so on, and the misdiagnosis rate is high [3]. Compared with CT and MRI, high-frequency ultrasound is convenient, fast and noninvasive for the diagnosis of pilomatricoma in the superficial area, with less cost and higher image resolution. It is a recommendable auxiliary diagnostic means. The main purpose of this study is to retrospectively analyze the high-frequency ultrasonographic data of 71 cases (77) of pilomatricoma in our hospital confirmed by ultrasonography and pathology, and summarize the characteristics of ultrasound images, so as to deepen people's understanding in the ultrasound image of pilomatricoma and improve the diagnosis rate.

\section{Data and Methods}

\subsection{General Data Collected}

From June 2013 to June 2017 in our hospital, 71 cases (77 cases) were diagnosed by high frequency ultrasonography and confirmed as pilomatricoma by pathology after operation, including 31 males and 40 females, with a male to female ratio of $1: 1.3$. The age of the patient was $1 \sim 68$ years old, with an average age of 27 years, of which 45 were less than 20 years of age, 16 were $20 \sim 40$ years old, and 10 more than 60 years old.

\subsection{Ultrasonic Instruments and Inspection Methods}

Philips iU22 ultrasound diagnostic apparatus, the frequency 
of $12 \sim 5 \mathrm{MHz}$. Making patients hold a comfortable posture, fully exposed to the lesion, then selected suitable ultrasound examination conditions, and scanned the mass in multiple directions after coating with a coupling agent. The location of the mass, the size of the tumor, the lump shape, the internal echo, the calcification, the liquefaction, the peripheral echo and the surrounding tissues were determined. Color Doppler ultrasound was used to observe the internal and peripheral blood flow.

\section{Results}

\subsection{Parts and Numbers}

62 cases occurred in the head and neck, accounting for $80.5 \%$ (including 19 in face, 17 in orbital, 11 in the neck, 9 in auricular, 6 in scalp), 11 in limbs, accounting for $14.3 \%$, and 4 in trunk. Among them, 66 patients were single, 4 had two lesions, and 1 had three lesions.

\subsection{Physical Examinations}

The skin color of the lesion: 49 were normal skin color, 17 local skin was dark red, and 11 were blue purple. Palpation in most cases is characterized by a clear border, adhesion and non-detachability with skin layer, but the basal part can be pushed up. Most of them are hard $(57,74 \%)$, and the remaining texture is medium.

\subsection{Ultrasound Sonographic Features}

The tumors are located subcutaneously, that is, between the skin layer and the subcutaneous fat layer. Of the tumors, 69 $(89.6 \%)$ were elliptical and $8(10.4 \%)$ were irregular. $67(87 \%)$ boundaries were clear, $6(7.8 \%)$ of the boundary was not clear, and $4(5.2 \%)$ was unable to define whether the boundary was clear because of serious rear attenuation. All cases were associated with different degrees of calcification on the basis of hypoechoic, of which $69(89.6 \%)$ were spot-like and patchy calcifications, and $8(10.4 \%)$ were coarse curved calcifications. $25(32.5 \%)$ blood flow signals were visible inside the tumor $(9$ of which were rich in blood flow signals, 16 were a few punctate blood flow signals), and 52 (67.5\%) tumors had no blood flow signals inside. Ultrasonographic images of typical cases were shown in Figure 1 and 2. The features of the sound image are given in Table 1.

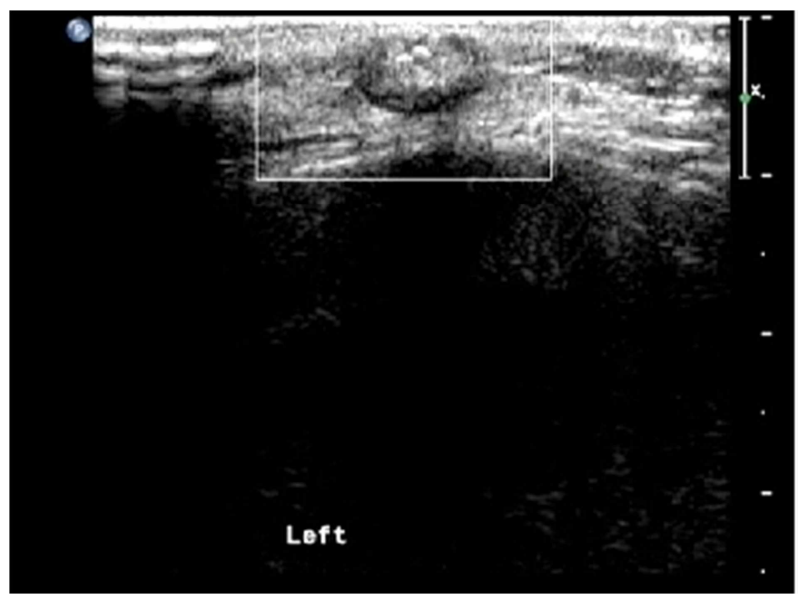

Figure 1. Internal patchy calcification, visible surrounding hypoechoic halo, no obvious blood flow signal.

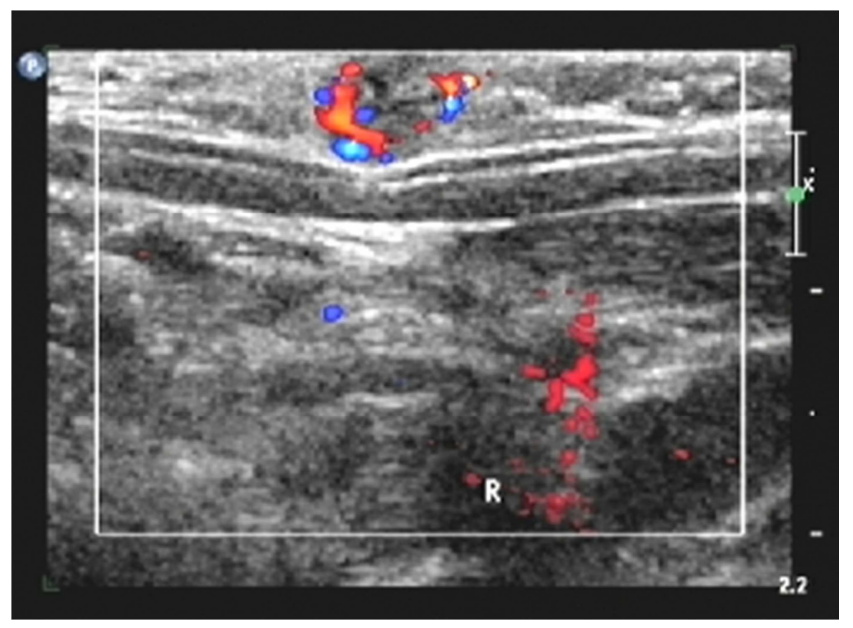

Figure 2. The boundary is not clear, and the blood flow signal is more abundant.

Table 1. Ultrasonographic features of hair mother tumor [n (\%)].

\begin{tabular}{llll}
\hline Characters of ultrasound images & Number of cases & Characters of ultrasound images & Number of cases \\
\hline Location & & low level echo & $35(45.5)$ \\
subcutaneous tissue & $77(100.0)$ & existent & $42(54.5)$ \\
Deep surface & $0(0.0)$ & Non-existent & $52(67.5)$ \\
Shape & & Blood flow signal \\
oval & $69(89.6)$ & Invisible & $25(32.5)$ \\
irregular & $8(10.4)$ & visible & $69(89.6)$ \\
boder & & calcification & $8(10.4)$ \\
clear & $67(87.0)$ & Punctate and flaky & $0(0.0)$ \\
Less clear & $6(7.8)$ & arc-shaped and coarse & Non-calcification \\
Hard to define & $4(5.2)$ & & \\
\hline
\end{tabular}

\subsection{Pathological Features}

Generally, most of the tumors have complete capsules. The cut is gray or dark brown. It is shaped like bean dregs, tough to the touch. Under the microscope, the tumor capsule is a fibrous connective tissue membrane. The tumor is composed of basophilic cells, shadow cells and transitional cells located between the two. The stroma of the tumor is composed of fibrous connective tissue, with focal calcification and giant cell reaction of the foreign body. The shadow cells are the key 
[4] for the diagnosis of pilomatricoma.

\section{Discussion}

Hair mother tumor is a benign nodular tumor located between the skin layer and the subcutaneous fat layer. In 1880, Malherbe and others first described it. In 1961, Forbis and Helwig suggested that it was named "pilomatricoma ". In 1974, WHO officially named it "pilomatricoma" and classified it as a hair follicle tumor. There are characteristic histopathological findings in pilomatricoma. Basophilic cells and shadow cells are important evidence for their diagnosis, and there are also transitional cells located between the two. Basophilic cells can differentiate into shadow cells, and can also be transformed into transitional cells. The latter becomes an amorphous matrix to be removed from the body. The proportion of basophils in the tumor is closely linked to the time of the tumorigenesis. If the time of tumor occurrence is short, the number of basophilic cells is pretty large. With the extension of time, the number of basophilic cells is decreasing, and the proportion of shadow cells is increasing. Calcification can be observed in most of the tumors. Calcification occurs mainly in the shadow cells, and can also exist in the interstitial [5]. The incidence of pilomatricoma is relatively low and can occur in all age groups, but is more common in children and adolescents [1, 6]. It is believed that the other peak of pilomatricom is after the age of 60 [7]. The incidence of women was a little bit higher than that of men. The proportion of men and women in this group was about 1: 1.3, which was consistent with the literature $[4,8]$. Most of the pilomatricom can occur at all sites that hair grows. The most frequent sites are the head and neck, the limbs and the body. Most of the cases in the head and neck can be seen in this study, about $80.5 \%$, consistent with the literature $[6,9]$. Most of the patients or patients' families unintentionally found that the patients themselves often have no obvious symptoms, and most of the swelling grow slowly. Only 13 of the cases in this group had local pressure pain, and there were no obvious symptoms in the remaining cases. Local skin can be a normal skin color, dark red or blue purple. When the skin is dark red or blue purple, it is easily misdiagnosed as a hemangioma in the clinic, and it needs to be identified at this time.

Calcification is the most important ultrasonic feature of the pilomatricoma. Calcification can be punctuated, patch shaped or arc-shaped, and its formation is the result of calcium deposition in the shadow cells, and the degree of calcification is related to the duration of hairy matrix tumors. In the early stage, the number of shadow cells in the tumor was small, and ultrasound showed punctate calcification. With the progression of disease, the number of shadow cells increased gradually. [10] Calcification became more obvious. It could be a patch or arc-shaped strong echo. In this group, most of the tumors were found punctate calcification, and 8 were curved rough calcification.

In the present study, hypoechoic halo was observed around 35 tumors and the pathology was confirmed by fibrous connective tissue. This sign could be used as another basis for ultrasound diagnosis, which can be used to be distinguished from other small surface nodules (such as dermoid cysts, sebaceous glands Cysts, etc.). There are still differences in the reports of blood supply in hair follicle tumors. In some literature, a rich blood flow signal [11] was found in the pilomatricoma. In this group, blood flow signals were observed in 25 masses, and 52 of them had no blood flow signals. [12] Therefore, color Doppler blood flow can be employed as an auxiliary diagnosis of pilomatricoma.

The pilomatricoma should be identified with an epidermoid cyst, hemangioma with vein stone, hemangioma with a thrombus, enlarged lymph node and hypodermic calcification. Epidermoid cysts usually have features of cystic change, such as rear echo enhancement, and no internal blood flow signals; hemangioma with phlebolith lesions will change in the probe after extrusion, and the blood flow signal will be more abundant; Lymph nodes are generally not single, if the lymph nodes with internal calcification are common in tuberculosis or tumor metastasis, the patient has a longer history. [13] In addition, it is necessary to exclude the skin metastasis of malignant tumors. The diagnosis of pilomatricoma is based primarily on histopathological examination. Ultrasonographic features of hair follicle tumor are valuable for the diagnosis of pilomatricoma, but the rate of misdiagnosis is still pretty high [3].

\section{Conclusion}

This study retrospectively analyzed the ultrasonographic data of 4 patients with pilomatricoma in our hospital for 4 years and concluded that the sonography of Hirschsprung's tumor has certain characteristics. When the clinical manifestations of the body surface (especially the head and neck) can promote subcutaneous solitary hard nodules, high-frequency ultrasonography prompted regular shapes, clear border and that there is a rough calcification in the interior and a hypoechoic halo around it. At this time, the possibility of pilomatricoma should be highly suspected. It is suggested that high-frequency ultrasound is of great significance in the diagnosis of hair mother tumor. However, due to the small sample size, the sonographic characteristics of the gross masses are limited. We need to further expand the sample size and conduct a multicenter joint study.

\section{References}

[1] Roche NA, Monstrey SJ, Matton GE. Pilomatricoma in children:common but often misdiagnosed [J]. Acta Chir Belg, 2010, 110 (2):250-254.

[2] Liu Hui, Lui Lui, Zhou Kesong, et al. Analysis of ultrasonic characteristics of hair mother tumor in [J]. clinical ultrasound medical journal, 2011, 13 (7):490-491.

[3] Wortsman X, Wortsman J, Arellano J, et a1. Pilomatricomas presenting as vascular tunlors on color Doppler ultrasound [J]. J Pediatr Surg, 2010, 45 (10):2094-2098. 
[4] Ieni A, Todaro P, Bonanno AM, et al. Limits of fine-needle aspiration cytology in diagnosing pilomatrixoma:a series of 25 cases with clinico-pathologic correlations $[\mathrm{J}]$. Indian J Dermatol, 2012, 57 (2):152-155.

[5] Xu Haihua, Huang Wei, Zuo Hailiang. Research progress of calcified epithelial tumor [J]. Medical Information 2015, 28 (27): 376 .

[6] Yang Lin, Luo Hong Hao. Analysis of ultrasonographic features of hair mother tumor [J]. Clinical ultrasound medical journal, 2016, 18 (3): 203-205.

[7] JULIAN CG, BOWERS PW. A clinical review of 209 pilomatricomas [J]. J Am, 1998, 39.

[8] Wan Jinzhou, Zhang Jingyi, He Ying, etc. 50 cases of high-frequency ultrasound features of hairy tumor [J]. Chongqing Medical, 2014, 43 (14): 1799-1801.
[9] Dong Zhihuai, Sun Yuefeng. Clinical analysis of 60 cases of children with hairy tumor [J]. Zhejiang Medical, 2012, 34 (15): 1299-1301.

[10] Liu Hao Tian, Ding Hongyu, Tao Meiying, et al. Ultrasound diagnosis and pathological analysis of tumor cells of the tumor [J]. Chinese Journal of Clinical Medicine (Electronic Version), 2013 (22): 188-190.

[11] Wortsman X, Wortsman J, Arellano J, et al. Pilomatricomas presenting as vascular tumors on color doppler ultrasound $[\mathrm{J}]$. Journal of Pediatric Surgery, 2010, 45 (10):2094-2098.

[12] Zhu Xiuling, Wang Jun, Li Jinying. Analysis of ultrasonic characteristics of 25 cases of hair mother tumor [J]. Southeast national defense medicine, 2017, 19 (5): 524-526.

[13] Zhang Jing, Bao Zuowei. Ultrasonic manifestations of cervical lymph node tuberculosis [J]. Traffic medicine, 2017, 31 (5): 480-481. 